\title{
Lower back pain; its causes and physical therapy: a review
}

\begin{abstract}
Low back pain (LBP) is one of the most frequent causes of disability. LBP is usually defined as pain localized below the margin of the last ribs (costal margin) and above the inferior gluteal lines, with or without lower limb pain. It has a prevalence of high during an individual's lifetime. At some point in life, some of adults have this syndrome. Most cases are nonspecific and occur in all age groups. There are a multitude of potential causes of acute lower back pain. The most common causes are thought to be muscle strains, ligament sprains, and tendonitis. Physical therapy such as fixation, thermotherapy, traction, and muscle strengthening, and guidance in regard to everyday activities plays an important role in the treatment of patients with low back pain. About half of patients with back pain receive physical therapy. It is found that both manual therapy and physical therapy had a positive effect on patients suffering from chronic back pain. The goal of the present study was to review the causes and physical therapy measures of patients with low back pain.
\end{abstract}

Volume 3 Issue 2 - 2018

\author{
Muhammad Ali,' Farouq S Nas² \\ 'Department of Microbiology, Kano University of Science and \\ Technology Wudil, Kano \\ ${ }^{2}$ Department of Biological Science, Bayero University, Nigeria
}

Correspondence: Muhammad Ali, Department of Microbiology, Kano University of Science and Technology Wudil, Nigeria, E-mail alimuhd4real@gmail.com

Received: March 19, 2018 | Published: March 29, 2018

Keywords: low back pain, physical therapy, fixation, thermotherapy, traction

\section{Introduction}

Low back pain (LBP) is one of the most frequent causes of disability. LBP is usually defined as pain localized below the margin of the last ribs (costal margin) and above the inferior gluteal lines, with or without lower limb pain. ${ }^{1}$ It has a prevalence of $60-85 \%$ during an individual's lifetime. At some point in life, between $15 \%$ and $20 \%$ of adults have this syndrome. Most cases (90\%) are nonspecific and occur in all age groups. ${ }^{2}$ Local sources of LBP are intervertebral discs, facet joints, sacroiliac joints, muscles, fascia, bones, nerves, and meninges. ${ }^{3}$ Causes of LBP are herniated discs, osteoarthritis, myofascial syndrome, spondylolisthesis, ankylosing spondylitis, rheumatoid arthritis, fibrosis, arachnoiditis, tumor, and infection. ${ }^{4}$ The number of spinal disorders is large, particularly those related to posture, inadequate body movements, and working conditions that may affect the spine. Due to the variety of factors involved, no therapeutic technique is effective for all patients. ${ }^{5}$ LBP is becoming an increasingly debilitating and costly problem. ${ }^{5,6}$ The cost of LBP is a heavy burden for the health care systems of many countries. ${ }^{7}$ Most persons will experience acute LBP during their lifetime. The first episode usually occurs between 20 and 40 years of age. For many, acute low back pain is the first reason to seek medical care as an adult. Pain can be moderate to severe and debilitating, causing anxiety. Many cases are self-limited and resolve with little intervention. However, 31 percent of persons with low back pain will not fully recover within six months, although most will improve. ${ }^{8}$ Recurrent LBP occurs in 25 to 62 percent of patients within one to two years, with up to 33 percent having moderate pain and 15 percent having severe pain. ${ }^{9}$ Acute LBP can be defined as six to 12 weeks of pain between the costal angles and gluteal folds that may radiate down one or both legs (sciatica). Acute LBP is often nonspecific and therefore cannot be attributed to a definite cause. However, possible causes of acute LBP (e.g., infection, tumor, osteoporosis, fracture, inflammatory arthritis) need to be considered based on the patient's history and physical examination. ${ }^{10,11}$ The goals of treatment for acute LBP are to relieve pain, improve function, reduce time away from work, and develop coping strategies through education. Optimizing treatment may minimize the development of chronic pain, which accounts for most of the health care costs related to low back pain. ${ }^{12}$ Physical therapy plays an important role in the treatment of patients with back pain. Between $10 \%$ and $50 \%$ of patients with back pain receive physical therapy. ${ }^{13-15}$ The effectiveness of physical therapy for patients suffering from back pain is however still unclear. ${ }^{14-18} \mathrm{In}$ a research made by ${ }^{16}$ it was that found that both manual therapy and physical therapy had a positive effect on patients suffering from chronic back pain (lasting for longer than six weeks). Jayson and Faas ${ }^{19,20}$ found that exercise therapy had no effect on patients with acute back pain.

\section{Anatomy of the lower back}

To understand various causes of LBP, it is important to appreciate the normal design (anatomy) of the tissues of this area of the body. Important structures of the lower back that can be related to symptoms in this region include the bony lumbar spine, intervertebral disc, ligaments around the spine and discs, spinal cord and nerves, muscles of the low back, internal organs of the pelvis and abdomen, and the skin covering the lumbar area. The bony lumbar spine is designed so that vertebrae "stacked" together can provide a movable support structure while also protecting the spinal cord from injury. The spinal cord is composed of nervous tissue that extends down the spinal column from the brain. Each vertebra has a spinous process, a bony prominence behind the spinal cord, which shields the cord's nervous tissue from impact trauma. Vertebrae also have a strong bony "body" (vertebral body) in front of the spinal cord to provide a platform suitable for weight bearing of all tissues above the buttocks. The lumbar vertebrae stack immediately atop the sacrum bone that is situated in between the buttocks. On each side, the sacrum meets the iliac bone of the pelvis to form the sacroiliac joints of the buttocks. ${ }^{21}$ The discs are pads that serve as "cushions" between the individual vertebral bodies. They help to minimize the impact of stress forces on the spinal column. Each disc is designed like a jelly donut with a central, softer component (nucleus pulposus) and a surrounding, firm outer ring (annulus fibrosus). The central portion of the disc is capable 
of rupturing (herniating as in a heniated disc) through the outer ring, causing irritation of adjacent nervous tissue and sciatica. Ligaments are strong fibrous soft tissues that firmly attach bones to bones. ${ }^{22}$ Ligaments attach each of the vertebrae to each other and surround each of the discs. The nerves that provide sensation and stimulate the muscles of the low back as well as the lower extremities (the thighs, legs, feet, and toes) all exit the lumbar spinal column through bony portals, each of which is called a "foramen." Many muscle groups that are responsible for flexing, extending, and rotating the waist, as well as moving the lower extremities, attach to the lumbar spine through tendon insertions. ${ }^{23}$

\section{Classification of low back pain}

There are 3 classes of LBP namely: Acute lower back pain which refers to lower back pain that lasts less than 4 weeks. Sub-acute lower back pain refers to lower back pain that lasts from 4 to 12 weeks, while chronic lower back pain is lower back pain lasting 12 weeks or longer. ${ }^{24}$

\section{Causes of lower back pain}

\section{Acute and sub-acute low back pain}

A minority of cases of back pain result from physical causes. Trauma to the back caused by a motor vehicle crash or a fall among young people and lesser traumas, osteoporosis with fractures, or prolonged corticosteroid use among older people are antecedents to back pain of known origin in most instances. ${ }^{25}$ Relatively less common vertebral infections and tumours or their metastases account for most of the remainder. Specific causes account for less than $20 \%$ of cases of back pain: the probability that a particular case of back pain has a specific cause is only $0.2 \%{ }^{25}$ So called "red flags", symptoms and signs that point to a specific cause and are well delineated in LBP initiative. ${ }^{26}$ Non-specific back pain is thus a major problem for diagnosis and treatment. Studies in the United Kingdom identified back pain as the most common cause of disability in young adults. ${ }^{27}$ the survey implicated back pain in more than 100 million work days lost per year. A survey in Sweden suggested that low back pain increased the number of work days lost from 7 million in 1980 to four times that (28 million) by 1987 however, social compensation systems might account for some of this increase. Jayson's group found that 35-37\% of workers experienced back pain in the month before their survey, with a peak in the incidence seen among those aged $49-59$ years. ${ }^{28}$

\section{Chronic back pain}

Psychological factors are even more important in people with chronic back pain. Dissatisfaction with a work situation, a supervisor, or a dead-end job and boredom contribute greatly to the onset and persistence of back pain As already mentioned, liberal compensation systems play a role in prolonging such pain not because of malingering, but rather because compensation leads to the now common perception that back pain is an injury. Curiously, it is classified thus in the industrial setting, in which workers' compensation systems or sickfunds come into play. ${ }^{29}$ Under the former WHO classification, back pain would be considered as a disability, and the social, design, and architectural barriers would be its handicaps..$^{30}$ Other activities often blamed, weight, lumbar lordosis, height, body mass index, and discrepancy between leg lengths may not play a major role. As stated, job dissatisfaction seems to be an important factor, but that, too, may reflect the pattern of reporting rather than actual causation. Disc herniation and spinal canal narrowing are so common as to be shown by imaging in most of the population in their later years, and in most cases, such conditions are not responsible for the pain. They often are cited as reasons for surgery, but only rarely are operations successful in alleviating the pain definitively. ${ }^{14}$

\section{Physical therapy for low back pain}

Physical therapy, which consists of exercise therapy, such as fixation, thermotherapy, traction, and muscle strengthening, and guidance in regard to everyday activities, contributes to reduction of LBP in either an auxiliary manner or as the fundamental modality of treatment, depending on the disease. Thus, although we recommend physical therapy, which is less invasive than drug therapy or surgical therapy, as the treatment of the first choice for LBP, it is important to determine whether its role is as auxiliary therapy or as the fundamental modality of treatment. ${ }^{31} \mathrm{~A}$ correct diagnosis of the disease responsible for the low back pain is also essential in order to make this distinction. Four different types of physical therapy for low back pain include;

\section{Fixation}

Fixation of the lower back by means of bleached cloth, corsets, etc., reduces low back pain by 3 actions: Limiting the movement of painful muscles, inter vertebral joints, inter vertebral discs, and fractured vertebral bodies. Maintaining good posture so as to reduce the mechanical load on the lower back, and reducing the mechanical load on the lower lumbar spine. ${ }^{31,32}$ Not only does limiting movement eliminate pain in the muscles, bones, joints, and inter vertebral discs that compose the lower back, but it can promote healing of the underlying disease. For example, acute muscular LBP caused by muscle and fascia tears, vertebral body compression fractures manifested by bone collapse, inter vertebral arthropathy manifested by mild inflammation in the joints, intervertebral disc hernia, in which a protruding disc compresses the spinal cord, etc. start to heal as a result of fixation. Methods in which the thorax and the pelvis are fixed rendering the lumbar spine rod-like is effective in limiting the movements of these tissues, and a slightly longer elastic corset than usual (Damen corset) or a Knight corset with a metal bar in its posterior portion are used for this purpose. ${ }^{31-33}$

\section{Thermotherapy}

Thermotherapy of low back pain is the most frequently used form of physical therapy performed in medical institutions. ${ }^{33}$ Thermotherapy is divided into methods that use surface heat and methods that use deep heat, and other forms of thermotherapy include hydrotherapy in the form of partial baths and whirlpool baths, and hot springs, which have a change of climate effect and mental-relaxation effect. There are 3 forms of thermotherapy that use surface heat, a form that uses conduction heat, including hot packs and paraffin baths, a form that uses radiant heat, including infrared rays, and a form that uses convection heat, including hydrotherapy and hot air baths. Methods that involve exposure to microwaves or ultra short waves, on the other hand, are classified as deep-heat thermotherapy. A therapeutic effect begins to occur after 3 to 5 minutes of exposure to their heat, and the maximal effect is obtained after 30 minutes. However, since burns occur when the temperature of the dermis and epidermis is raised to $42^{\circ} \mathrm{C}$ and heated for several hours, thermotherapy at no more than $45^{\circ} \mathrm{C}$ for about 30 minutes can be said to be in the safe range (but there are individual differences). 


\section{Traction}

Traction is frequently performed in diseases of the cervical spine and for fracture reduction and fixation. However, because of the efficacy of anti-inflammatory analgesics, blocking therapy and the development of surgical procedures that replace it, traction is being used less often to eliminate LBP. The recent trend toward shorter stays in medical institutions can be cited as another reason why traction for LBP has been replaced by other treatment modalities. Traction exerts its greatest efficacy against LBP when used to treat lumbar intervertebral disc herniation. A pelvic traction band is usually wrapped around the pelvis with the patient resting in the supine position, and traction is applied by means of a $5-10 \mathrm{~kg}$ weight at the foot of the bed. This procedure is effective, because when the lumbar area-pelvis is flexed 20-30 degrees like a craft knife, it acts in a direction that reduces the protruding intervertebral disc back into position. Because of the strong traction toward the foot of the bed, the body imperceptibly slides in that direction and the weight exerting the traction force may eventually touch the floor. ${ }^{33}$ To prevent that from happening, the head of the bed can be lowered slightly and the bottom raised by placing blocks under the legs at the foot of the bed and raising it $10 \mathrm{~cm}$ off the floor. When traction is applied in this manner, the symptoms improve within 3-7 days. Resolution of the LBP is often observed in about 2 weeks, and the traction can be discontinued at that time. The traction used for lumbar intervertebral disc hernias, called "pelvic traction", used to be performed for lumbar spinal canal stenosis, which was diagnosed as sciatica, and was effective.

\section{Muscle strengthening (exercise)}

Exercise therapy relieves LBP in chronic muscular lower back pain and degenerative diseases of the lumbar spine by strengthening the lumbar muscles and abdominal muscles. Lumbar muscles whose muscle strength has been increased by exercise are able to lifting the upper body, with the lower back acting as the pivot. ${ }^{32,33}$ The abdominal muscles act as a "natural" corset, they contribute to raising the pressure in the abdominal cavity and reducing the load of the force on the lower back. Two simplified Williams' exercises and stretch exercises performed before and after them appear to be useful for this purpose. Eliminating the movements of other muscles by slightly flexing the knees and slowly lifting the upper portion of the body by contracting the abdominal muscles alone is an effective means of strengthening the abdominal muscles. However, it is sometimes difficult to perform unless patients have regularly trained their abdominal muscles, and excessive repetitive abdominal strengthening exercises sometimes cause such intense pain the following day that the patient seems to have peritonitis. Because of this it is advisable to gradually strengthen the muscles by using techniques and numbers of repetitions tailored to each individual's physical constitution. In regard to the lumbar muscles, there is a tendency to be kyphotic in osteoporosis and scoliosis is sometimes present in cases of intervertebral disc degeneration, making it difficult to bend the body back, and forcing the back into retroflexion may risk exacerbating the lower back pain. Keeping these points in mind, LBP is often relieved by about 3-4 weeks after starting 20-30 repetitions of abdominal muscle and lower back muscle strengthening exercises a day. ${ }^{31}$ Before and after the training exercises it is useful to perform lower back rotation exercises in the same supine position by lowering the flexed knees to the right and left and stretching and moving the muscles and ligaments of the lower back and buttocks. In the low back pain prevention exercise class in Tokyo that the author was involved in, practical training guidance centered on the exercises was provided for 10 weeks with the aim of establishing regular exercise habits at home. The result was improvement of the low LBP in 32 of the 50 participants (48 women, 2 men; mean age 53.8 years), and measurements of several parameters in the patients who showed improvement revealed that the changes were significant. ${ }^{31}$ Exercise therapy is the sole educational form of physical therapy for LBP, and it demands an effort on the part of patients, but if patients can learn muscle strengthening and make it a regular habit, it is a "good medicine" that they can take with them wherever they go. ${ }^{33}$

\section{Conclusion}

At some point in life, between $15 \%$ and $20 \%$ of adults have this syndrome. Most cases $(90 \%)$ are nonspecific and occur in all age groups. There are a multitude of potential causes of acute lower back pain. The most common causes are thought to be muscle strains, ligament sprains, and tendonitis. Physical therapy such as fixation, thermotherapy, traction, and muscle strengthening, and guidance in regard to everyday activities plays an important role in the treatment of patients with back pain. It is found that both manual therapy and physical therapy had a positive effect on patients suffering from chronic back pain.

\section{Acknowledgements}

The authors wish to acknowledge the contribution of Dr. AA Minjibir of Department of Pharmaceutical Technology, School of Technology Kano for his suggestions and corrections.

\section{Conflict of interest}

The authors declared that no conflict of interest exists.

\section{References}

1. Van Middelkoop M, Rubinstein SM, Verhagen AP, et al. Exercise therapy for chronic nonspecific low-back pain. Best Pract Res Clin Rheumatol. 2010;24(2):193-204

2. Krismer M, Van Tulder M. Strategies for prevention and management of musculoskeletal conditions. Low back pain (non-specific). Best Pract Res Clin Rheumatol. 2007;(21):77-91.

3. Winkelstein BA, Weinstein JN, De Leo JA. The role of mechanical deformation in lumbar radiculopathy: an in vivo model. Spine. 2002;27(1):27-33

4. Verbeek JH, Vander Weide WE, Van Dijk FJ. Early occupational health management of patients with back pain: a randomized controlled trial. Spine. 2002;27(17):1844-1851.

5. Mikhail C, Korner-Bitensky N, Rossignol M, et al. Physical therapist's use of interventions with high evidence of effectiveness in the management of a hypothetical typical patient with acute low back pain. Physical Therapy. 2005;85:115-167.

6. Mannion F, Junge A, Tarmela S, et al. Part 3 Factors influencing Selfrated Disability and its change following Therapy. Spine. 2001;(26):920 929.

7. Linton SJ. The socio-economic impact of chronic back pain: is anyone benefiting. Spain. 1998;(75):163-168.

8. Carey TS, Garrett J, Jackman A, et al. The outcomes and costs of care for acute low back pain among patients seen by primary care practitioners, chiropractors, and orthopedic surgeons. The North Carolina Back Pain Project. N Engl J Med. 1995;333(14):913-917. 
9. Stanton TR, Henschke N, Maher CG, et al. After an episode of acute low back pain, recurrence is unpredictable and not as common as previously thought. Spine. 2008;33(26):2923-2928.

10. Institute for Clinical Systems Improvement (ICSI). Adult low back pain (guideline). 2010.

11. McIntosh G, Hall H. Clinical Evidence. Low back pain (acute). 2011.

12. Becker A, Held H, Redaelli M, et al. Low back pain in primary care costs of care and prediction of future health care utilization. Spine. 2010;35(18):1714-1720.

13. Biering-Seresen. A prospective study of low back pain in General population. Scand J Rehabil Med. 1983;(15):89-96.

14. Deyo RA, Haselkorn J, Hoffman R, et al. Designing studies of diagnostic tests for low back pain and inflammatory mediators. Spine. 1994;(20):59-68.

15. Davis PC, Wippold FJ, Brunberg JA, et al. American College of Radiology ACR appropriateness criteria. Low back pain. 2011.

16. Koes BW, Van Tulder MW, Thomas S. Diagnosis and treatment of low back pain. British Medical Journal. 2006;(332):1430-1434.

17. Frank A. 'Low back pain'. British medical journal. 1993;(306):901-909.

18. Fritz JM, Cleland JA, Speckman M, et al. Physical therapy for acute low back pain: associations with subsequent healthcare costs. Spine. 2008;33(16):1800-1805.

19. Jayson MIV. Why does acute back pain become chronic? Chronic back pain is not acute back pain lasting longer. British Medical Journal. 1997;(314):1639-1640.

20. Faas A. Oefenfherape MJ mute Lag8 Rugpiin: €en lnterventieonderzoek BIJ 40 Huisertsprektijken, Offsetdruk wemaal. oldenzeal. 1992.

21. Acosta F, Galvez L, Aryan H, et al. Recent Advances: Infections of The Spine. Curr Infect Dis Rep. 2006;8(5):390-393.

22. Brian A, Casazza M. Diagnosis and Treatment of Acute Low Back Pain American Family Physician. 2012;85(4):343-350.

23. Boden SD, Davis DO, Dina TS, et al. Abnormal Magnetic-Resonance Scans Of The Lumbar Spine In Asymptomatic Subjects. A Prospective Investigation. J Bone Joint Surg Am. 1990;72(3):403-408.
24. Nachemson AL, Waddell G, Norlund A. Epidemiology of neck and low back pain. In: Neck and back pain: the scientific evidence of causes, diagnosis and treatment. Philadelphia (PA): Lippincott Williams \& Wilkins; 2010

25. Bigos SJ, Bowyer O, Braea G, et al. Acute low back pain problems in adults. Clinical practice guideline no. 14. AHCPR Publication No. 950642. Rockville (MD): US Department of Health and Human Services; 1994.

26. Ehrlich GE, Khaltaev NG. Low back pain initiative. Geneva. World Health Organization; 1994.

27. Croft P, Rigby AS, Boswell R, et al. The prevalence and characteristics of chronic widespread pain in the general population. J Rheumato. 1993;(20):710-713.

28. Papageorgiou A, Croft P, Thomas E, et al. Influence of previous pain experience on the episodic incidence of low back pain. Results from the South Manchester back pain study. Pain. 1996;(66):181-185.

29. Hadler NM. Occupational musculoskeletal disorders. Philadelphia (PA) Lippincott Williams and Wilkins. 2018;57(6).

30. International classification of impairments, disabilities, and handicaps. Geneva. World Health Organization. 1999.

31. Iwakura $H$, Watanabe $H$, Kakurai S. Diseases of the Trunk and Musculoskeletal System, and Rehabilitation, Ishiyaku Publishers; 1985:133-196.

32. Ishigami S. Indications and selection of physical therapy for pain (with special reference to thermotherapy). Itami to Rinsh. 2001;(1):417-422.

33. Ishiyama Y, Minami S, Hayashi Y. Usefulness of exercise to prevent low back pain with the objective of promoting the health of the middle aged and elderly. Journal of Clinical a Sports Medicine. 1993;(10):15331539. 